\title{
Chemical Nanoarrays for Early Detection and Screening of Lung Cancer via Volatile Biomarkers
}

\author{
H. Haick $^{* 1}$, O. Barash ${ }^{1}$, M. Hakim ${ }^{1}$, U. Tisch ${ }^{1}$, Radu lonescu', Maya llouze ${ }^{3}$, Paul A. Bunn Jr. R. ${ }^{2}$, Jane \\ Mattei ${ }^{2}$, York E. Miller ${ }^{2}$, Timothy C Kennedy ${ }^{2}$, John D Mitchell', Michael J. Weyant ${ }^{2}$, \\ F. R. Hirsch ${ }^{2}$, N. Peled $d^{2,3}$ \\ ${ }^{1}$ Department of Chemical Engineering and Russell Berrie Nanotechnology Institute, Technion - Israel \\ Institute of Technology, Haifa 32000, Israel. Email: hhossam@tx.technion.ac.il \\ ${ }^{2}$ University of Colorado Cancer Center, Divisions of Pulmonary Sciences and Critical Care and \\ Medical Oncology, UC Denver, Aurora, Colorado 80045, United States \\ ${ }^{3}$ The Thoracic Cancer Research and Detection Center, Sheba Medical Center, Tel Aviv University,
} Tel-Aviv 52621, Israel

\begin{abstract}
:
Lung cancer is the leading cause of cancer-related deaths worldwide. The cancer's stage, histology and genetic mutations determine a patient's prognosis and treatment. Here we present an artificial electronic nose (NA-NOSE), which makes use of cross-reactive chemical sensor nanoarrays, for the detection of volatile biomarkers in breath samples as well as in the headspace of in-vitro cell lines of lung cancer. A series of proof-of-concept studies with the NA-NOSE have shown an excellent ability to distinguish between lung cancer and healthy states, between different histologies of link cancer, and between lung cancer genetic mutations that can benefit from targeted treatments. The proposed biomarker-based testing NA-NOSE technology holds future potential as a cost-effective, fast and reliable diagnostic test for early disease detection and monitoring of the disease progression. The NANOSE would be suitable for use outside of specialist settings and could significantly reduce in the burden on the health budget.
\end{abstract}

Key words: Lung Cancer, Volatile Biomarkers, Breath Testing, Headspace Sampling, Sensor, Nanoarray

\section{Background}

Lung cancer (LC) is the leading cause of cancer mortality with more than 1 million deaths worldwide every year.[1,2] Cancer emergence, aggressiveness and treatment response varies greatly from patient to patient.[3] The cancer's stage, histology and genetic mutations are important factors when determining a patient's prognosis and deciding which treatment would be optimal in an individual case.[4-7] Conventional detection methods involve invasive tissue sampling from the tumor, and frequent monitoring is needed to detect changes in the cancer cells over time. An emerging approach for the comprehensive diagnosis of LC (including stage, histology and genetic mutations) relies on volatile biomarkers that are emitted from cell membranes. These volatile biomarkers can be detected either directly from the headspace of the cancer cells or via the exhaled breath. We have developed and tested a highly-sensitive, inexpensive, fast and non-invasive tool for the detection of (pre)neoplastic volatile biomarkers. This tool is based on an artificial electronic nose (NANOSE), which makes use of cross-reactive chemical sensor nanoarrays (based on chemiresistive layers of organically stabilized gold nanoparticles) that are trained in their ensemble to detect cancer biomarkers. The nanoarray concept was applied to both breath testing, using collected breath samples from lung cancer patients and various control groups, and to headspace sampling from lung cancer cell lines.

\section{Methods}

Breath samples were collected from patient cohorts having non-small-cell LC (NSCLC) or small cell LC (SCLC), as described elsewhere. The patients were diagnosed and staged using conventional (invasive) methods. [3,4] Headspace samples were collected from SCLC and NSCLC cell lines, as described 
elsewhere.[7-9] The NSCLC cell lines had welldefined genetic mutations. The cell lines were analyzed using the NA-NOSE system in conjunction with pattern recognition methods.

\section{Identification of LC Patients}

LC shares many risk factors with other types of cancer (e.g. advanced age, tobacco consumption etc.).[1,2] It is therefore important to distinguish LC from other types of cancer.

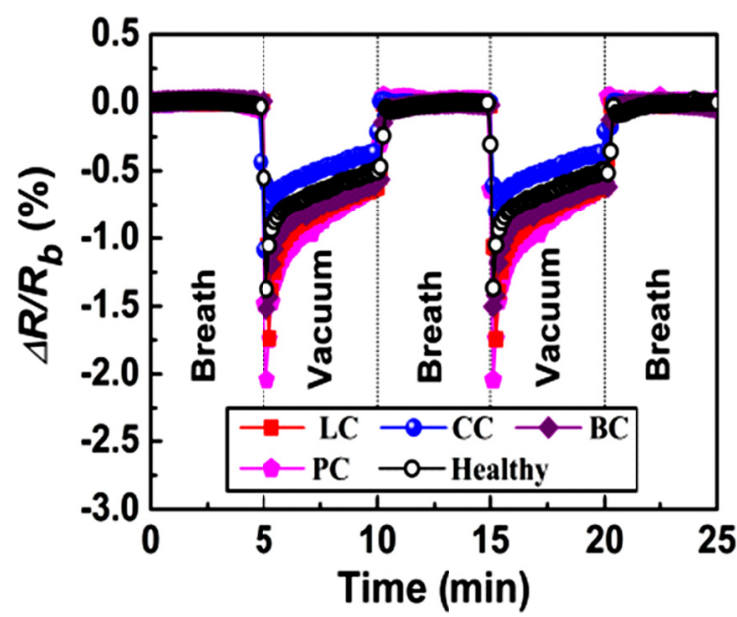

Clinical studies on breath samples collected from 177 volunteers demonstrated the discriminative power of the NA-NOSE breath test to distinguish LC patients from healthy controls and from patients having colon cancer (CC), breast cancer (BC) or prostate cancer (PC) with $>95 \%$ diagnostic accuracy (see Figure 1).[3] The NA-NOSE distinguished between the breath of cancer patients and that

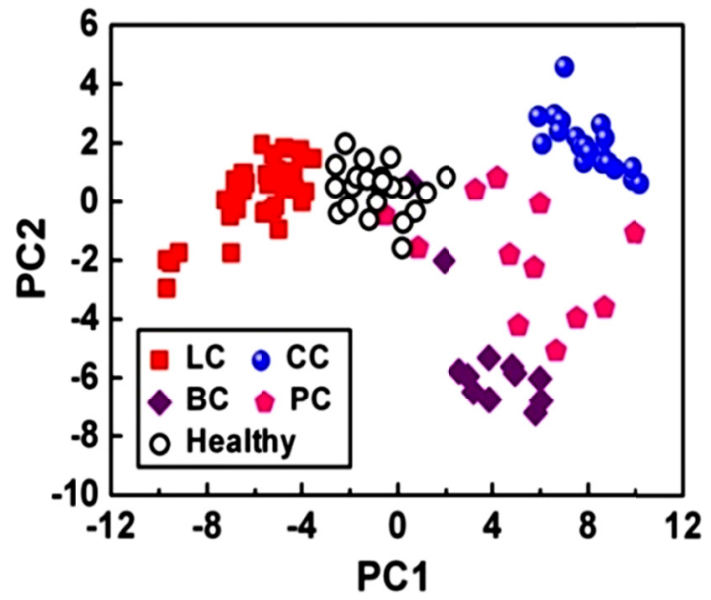

Fig. 1. (Left): Representative response of a NA-NOSE sensors array to breath of patients with LC, CC, BC and $P C$, as well as healthy controls; (Right): PCA map showing the separation between the five test groups.[3]

of healthy individuals with high accuracy and in the presence of confounding factors (e.g., age, gender, ethnic origin, family cancer history, intake of food additives, drug treatment, exposure to environmental toxins and smoking habits, etc.). The unambiguous detection of LC within a mixed population of healthy persons and persons suffering from the four most widespread cancers in a single breath test could form the basis of a future cancer screening test for the general population. In a complementary study we showed that the NANOSE could identify and distinguish specifically between LC and head-and-neck cancer (HNC) that are both closely related to tobacco smoking, and, therefore occur in the same population.[4] The results showed excellent separation between LC patients, HNC patients and healthy controls.

\section{Determination of the LC Stage and Histology} NSCLC and SCLC are the most prevalent histological types, representing approximately $80 \%$ and $15 \%$ of LC, respectively. Both LC types occur in a similar population of heavy smokers, but require different disease management. Surgery is performed on many of the NSCLC patients (stages I, II and some of IIIA), while chemotherapy and radiotherapy are the backbone of SCLC treatment, except in the very early stage (IA).[5] Early-stage LC (stage III) is often curable, but prognosis is grave and recurrence rates are high for advanced disease (stages III and IV).[6]

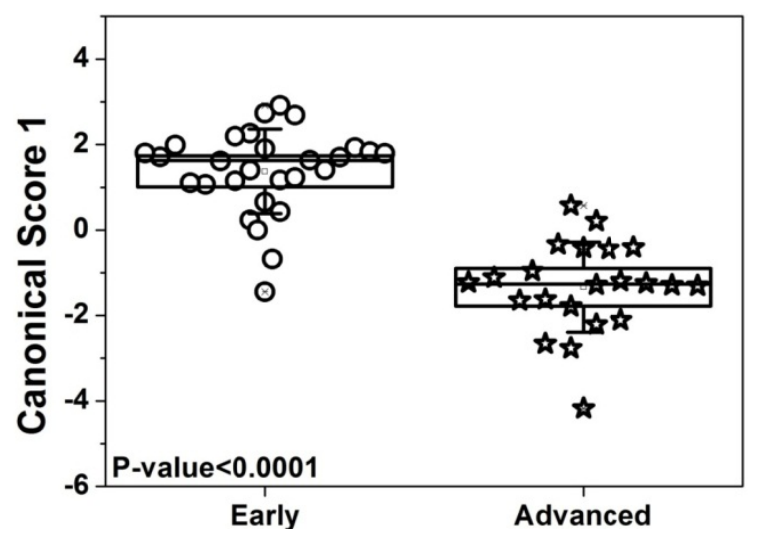

Fig. 2. Graphical representation of the first canonical score values for breath samples of patients with early stage (stages I\&II) or advanced (stages III\&IV) NSCLC, using a NA-NOSE system. Each point represents one patient. The positions of the canonical score mean values are marked with $\square$, the boxes correspond to their 95\% confidence limits, and the error bars corresponds to the standard deviation of the first canonical score values. The confidence intervals are significantly separated ( $p$ values $<0.0001$ by Wilcoxon test). 
The feasibility of the NA-NOSE concept for LC staging and for histological sub-typing was shown in a separate clinical study, using breath samples from 74 patients with solitary pulmonary nodules (SPNs).[7] Excellent separation could be achieved between benign vs. malignant SPNs $(p<0.0001$; accuracy $88 \pm 2 \%$ ), between the histological sub-types SCLC and NSCLC $(p=0.0015$; accuracy: $94 \pm 1 \%$ ) and between early stage and advanced disease ( $p<0.0001$; accuracy: $88 \pm 2 \%$; see Figure 2).

A series of complementary in-vitro studies have confirmed the potential of the NA-NOSE concept for LC sub-typing.[8,9] The NA-NOSE clearly distinguished headspace samples from NSCLC and SCLC cell lines. Furthermore, excellent separation could be achieved between two common sub-types of NSCLC (adenocarcinoma and squamous cell carcinoma), which require different disease management.[8]

\section{Genetic Profiling of Cancer}

Gene expression profiling [10] plays a key role in accurately classifying tumors of individual patients, in predicting the response to the available treatments and in personalizing cancer treatment [11-14].

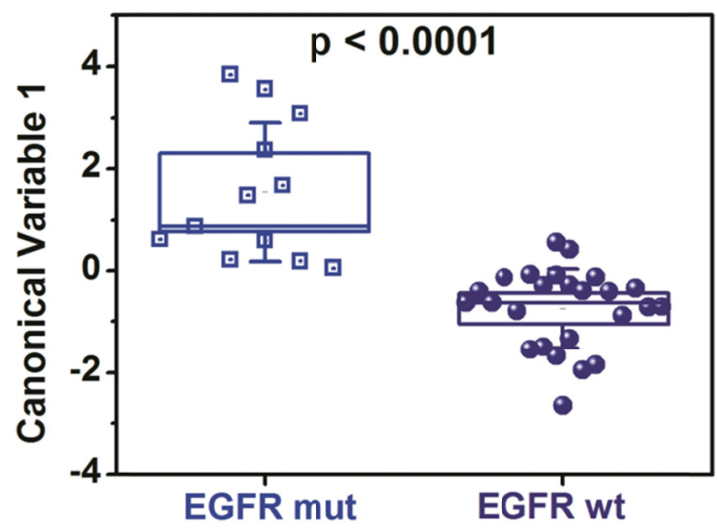

Fig. 3. DFA plots of the first canonical variable that was calculated from the NA-NOSE responses to the headspace samples of NSCLC cell lines having EGFR mutations. The EGFR wt samples include cell lines with KRAS mutations, ALK fusion and genetic mutations that are wt to all three studied mutations.

We have explored the applicability of the NANOSE concept for gene expression profiling in a further in-vitro study. Headspace samples of several cell lines with specific well-defined genetic mutations (10 with epidermal growth factor receptor (EGFR) mutations;[13] 14 with V-Ki-ras2 Kirsten rat sarcoma viral oncogene homolog (KRAS) mutations; 8 with anaplastic lymphoma kinase (ALK) fusion) were analyzed and compared. A group of five samples from LC cell lines with genetic mutations that are wild type (wt) compared to the three mutations of interest was included as additional control group. The NA-NOSE could clearly identify patterns of volatile biomarkers emitted from cell lines that exhibited EGFR and KRAS mutations, and ALK fusion of the LC cells. Figure 3 shows that the LC cells with EGFR mutations could clearly be distinguished from the LC cells with other genetic mutations. A leave-one-out cross validation procedure was performed and yielded a classification accuracy of $92 \%$ for EGFR mutations. Similar results were obtained for the cells having KRAS mutations and ALK fusion.

\section{Conclusions}

The NA-NOSE concept has the potential to reduce cancer mortality allowing easy, inexpensive and fast detection of the cancer subtype and/or cancer related genetic mutations that can benefit from oral targeted therapy (e.g. EGFR tyrosin kinase inhibitors). The easy-to-use NA-NOSE technology detects cancer and identifies cancer stage, histology, and cancer-related genetic mutations based on a change in the blood chemistry and/or metabolic activity rather than by tumor imaging, thus permitting earliest cancer detection i.e. before a tumor of detectable size has formed, and/or assists the swift choice of the therapeutic approach.

\section{Acknowledgements}

The research leading to these results has received funding from the FP7-Health Program under the LCAOS (grant agreement no. 258868; H.H. and N.P.), FP7's ERC grant under DIAG-CANCER (grant agreement no. 256639; H.H.), the IASLC (N.P.) and the Fulbright Israel-US foundation (N.P.).

\section{References}

[1] Cutler DM. Are we finally winning the war on cancer? J Econ Perspect, 22, 3-26 (2008).

[2] Jemal A, Siegel R, Xu J, Ward E. Cancer statistics, 2010. CA Cancer J Clin, 60, 277-300 (2010).

[3] Peng G, Hakim M, Broza YY et al. Detection of lung, breast, colorectal, and prostate cancers from exhaled breath using a single array of nanosensors. Br. J. Cancer, 103, 542-551 (2010).

[4] Hakim M, Billan S, Tisch $U$ et al. Diagnosis of head-and-neck cancer from exhaled breath. $\mathrm{Br}$. J. Cancer, 104, 1649-1655 (2011). 
[5] Vallieres E, Shepherd FA, Crowley J et al. The IASLC Lung Cancer Staging Project: proposals regarding the relevance of TNM in the pathologic staging of small cell lung cancer in the forthcoming (seventh) edition of the TNM classification for lung cancer. J Thorac Oncol, 4, 1049-1059 (2009).

[6] Goldstraw P, Crowley J, Chansky $\mathrm{K}$ et al. The IASLC lung cancer staging project: proposals for the revision of the TNM stage groupings in the forthcoming (seventh) edition of the TNM classification of malignant tumours. Journal of Thoracic Oncology, 2, 706-714 (2007).

[7] Peled N, Hakim M, R. PABJ et al. Breath analysis in the post NLST era: distinguishing malignant from benign pulmonary nodules. (To be published).

[8] Barash O, Peled N, Tisch U, Bunn PA, Hirsch FR, Haick $\mathrm{H}$. Classification of lung cancer histology by gold nanoparticle sensors. Nanomedicine, Epub ahead of print available at: http://dx.doi.org/10.1016/j.nano.2011.10.001 (2012).

[9] Barash O, Peled N, Hirsch FR, Haick H. Sniffing the unique "odor print" of non-small-cell lung cancer with gold nanoparticles. Small, 5, 26182624 (2009).

[10] O'Callaghan DS, Savale L, Montani D et al. Treatment of pulmonary arterial hypertension with targeted therapies. Nature Rev. Cardiol., 8, 526538 (2011).

[11] Shedden K, Taylor JMG, Enkemann SA et al. Gene expression-based survival prediction in lung adenocarcinoma: a multi-site, blinded validation study. Nature Med., 14, 822-827 (2008).

[12] Einhorn LH. Will we ever have personalized medicine for non-small cell lung cancer? J. Thorac. Oncol., 1, 737-739 (2006).

[13] Merlo V, Longo M, Novello S, Scagliotti GV. EGFR pathway in advanced non-small cell lung cancer. Front Biosci., 3, 501-517 (2011).

[14] Kato Y, Peled N, Wynes MW et al. Novel epidermal growth factor receptor mutationspecific antibodies for non-small cell lung cancer: Immunohistochemistry as a possible screening method for epidermal growth factor receptor mutations. J. Thorac. Oncol., 5, 1551-1558 (2010). 